\title{
Effects of Total Dietary Fiber on Cecal Microbial Community and Intestinal Morphology of Growing White Pekin Duck
}

\author{
Yongsheng Hao, Zhanqing Ji, Zhongjian Shen, Yongbao Wu, Bo Zhang, Jing Tang, \\ Shuisheng Hou and Ming Xie* \\ State Key Laboratory of Animal Nutrition, Institute of Animal Science, Chinese Academy of Agricultural Sciences, Beijing, \\ China
}

OPEN ACCESS

Edited by:

Miguel Gueimonde,

Institute of Dairy Products of Asturias

(IPLA), Spain

Reviewed by:

Qiufeng Zeng,

Sichuan Agricultural University, China

Jose Luis Alonso,

University of Vigo, Spain

*Correspondence:

Ming Xie

caasxm@163.com

Specialty section:

This article was submitted to

Microorganisms in Vertebrate

Digestive Systems,

a section of the journa

Frontiers in Microbiology

Received: 18 June 2021

Accepted: 04 August 2021

Published: 01 September 2021

Citation:

Hao Y, Ji Z, Shen Z, Wu Y,

Zhang B, Tang J, Hou S and Xie M (2021) Effects of Total Dietary Fiber on

Cecal Microbial Community and Intestinal Morphology of Growing White Pekin Duck.

Front. Microbiol. 12:727200 doi: 10.3389/fmicb.2021.727200
The current study was to investigate the effects of total dietary fiber (TDF) on growth performance, cecal structure, cecal microbial community, and short-chain fatty acids (SCFAs) profiles in the cecum of growing White Pekin ducks. A total of 108 male Pekin ducks of 14-days-old were randomly allocated and fed diets containing 12.4, 14.7, and $16.2 \%$ TDF for 35 days. Each dietary treatment consisted of six replicates with six birds each. The results showed that 14.7 and 16.2\% TDF treatments promoted growth performance relative to $12.4 \%$ TDF treatments $(P<0.05)$. A total of 14.7 and $16.2 \%$ TDF treatments significantly elevated villus height, the ratio of villus height to crypt depth and muscle layer thickness of cecum, and lowered crypt depth compared with 12.4\% TDF treatment $(P<0.05)$. Simultaneously, 14.7 and $16.2 \%$ TDF treatments up-regulated Claudin-1 mRNA expression of barrier genes in the cecum compared with 12.4\% TDF $(P<0.05)$. Butyrate-producing bacteria like Oscillopiraceae affiliating to the phyla Firmicutes were observed as a biomarker in the 16.2\% TDF. Higher concentration of butyrate in the cecum was obtained in the 14.7\% TDF compared with 12.4 and $16.2 \% \operatorname{TDF}(P<0.05)$. The concentrations of isobutyrate, valerate, and isovalerate in the cecum were significantly increased in the 16.2\% TDF compared with 12.4 and $14.7 \%$ TDF $(P<0.05)$. Meanwhile, the abundance of genus UCG-005 and Enterococcus was positive correlations with isobutyrate and valerate $(P<0.05)$. However, the concentration of propionate in the cecum significantly decreased in 14.7 and $16.2 \%$ TDF treatments relative to $12.4 \%$ TDF treatments $(P<0.05)$. In summary, increasing TDF levels improved growth performance, cecal histomorphology, and barrier function of meat ducks and it might be mediated by the changes of microbiota communities, especially bloom of SCFAs-producing bacteria, which facilitated the interaction between intestinal mucosa and microbiota.

Keywords: total dietary fiber, ducks, microbiota, short-chain fatty acids, barrier function 


\section{INTRODUCTION}

Nowadays, use of antibiotics has been banned in some countries and gut barrier health is becoming a potential risk for poultry production. Fortunately, the beneficent role of fibers in modulating gut microbiome, stimulating immunity, and promoting gut integrity is noticed (Singh and Kim, 2021; Tejeda and Kim, 2021) and inclusion of fibers in poultry diets is becoming a nutritional strategy to protect poultry gut from invasion of pathogen and toxins (Kheravii et al., 2018), although the fiber contents from feedstuffs had lowering-digestibility and antinutrient properties. Likes other avian species, dietary fiber was also beneficial to duck growth and gut health and ducks could adapt to a wide dietary crude fiber range from 3.09 to $7.52 \%$ (Han et al., 2017). Furthermore, ducks fed with high dietary crude fiber levels had increased gizzard development, jejunal morphology, energy retention, excreta nutrients availability, and standardized ileal digestibility of amino acids, and high dietary fiber also alleviated hepatic fat deposition via inhibiting lipogenic gene expression in meat ducks (Han et al., 2017; Qin et al., 2018).

In the past decades, the concept of crude fiber has still been commonly used in poultry diet formulation, but this concept may be questioned because non-starch polysaccharides and resistant starch are usually ignored by this concept, and these ignored fibrous compounds play a crucial role in intestinal functioning, nutrient digestion, and intestinal microflora modulation. At the same crude fiber levels, the starter broilers fed fiber from soy hulls had better growth performance, intestinal histomorphology, and nutrient digestibility compared with the birds fed fiber from purified cellulose (Tejeda and Kim, 2020). Recently, when ducks were fed with diets containing high levels of resistant starch, the gut barrier was improved by enhancing intestinal morphology and barrier markers expression, modulating the microbiota composition, and attenuating inflammatory markers (Qin et al., 2019, 2020). In the aforementioned two studies, soy hulls contained many fibrous components out of category of crude fiber, and resistant starch was out of category of crude fiber. Fortunately, compared with the concept of crude fiber, the more fiber fractions including non-starch polysaccharides and resistant starch were covered by the concept of total dietary fiber (TDF) developed by the Association of Official Analytical Chemists, AOAC International, 2007, and thus the concept of total crude fiber may be preferable to the concept of crude fiber when the relationship between dietary fiber and gut health was emphasized.

Recently, the association between microbial community and ileal gene expression on intestinal wall thickness alterations in chickens revealed that well-developed intestinal morphology could increase the abundance of beneficial bacteria coupled with active community anabolism, thus enhancing the absorption and immune function of intestinal epithelial cells (Tang et al., 2020). Furthermore, the microbial community and short-chain fatty acids (SCFAs) mapping in the intestinal tract of quail showed that cecum was the core location of fiber fermentation and SCFAs production because cecum has the most total bacteria population and highest SCFAs concentrations among all intestinal segments
(Du et al., 2020). When resistant starch was fed to ducks, the ducks with improved cecal morphology also had enhanced the abundance of SCFAs-producing bacteria in the cecum (Qin et al., 2020). In pigs, the positive effects of dietary fiber on intestinal barrier function also could be explained by improving distal gut morphology and altering microbiota composition, especially enhancing the abundance of butyrate-producing bacteria (Chen et al., 2013). Recently, the concept of TDF was used in pig diet formulation, and appropriate TDF level could increase the diversity and metabolic capacity of cecal microbiota to improve the utilization efficiency of fiber resources without altering the growth rate of pigs (Pu et al., 2020). However, in ducks, the researches on the potential beneficial effects of TDF on growth performance and gut health were still limited, and it was not clear for gut-microbiota interplay at high fiber intake. Therefore, the concept of TDF was utilized in the present study and the objective of our study was to discuss the effects of TDF on growth performance, cecal histomorphology, and cecal microbiota of growing Pekin ducks.

\section{MATERIALS AND METHODS}

\section{Experimental Design and Bird Management}

The dose-response experiment with three TDF levels (12.4, 14.7, and $16.2 \%)$ was conducted with 14-days-old male White Pekin ducks. A total of 120 one-day-old male white Pekin ducklings from one commercial hatchery were fed with a commercial starter diet containing $12.12 \mathrm{MJ}$ metabolizable energy $/ \mathrm{kg}$ and $200 \mathrm{~g}$ crude protein $/ \mathrm{kg}$ of diet until 14 days of age. At 14 days of age, all birds were weighed individually and the birds with the lowest or highest body weight were removed, and 108 birds were selected from the remaining birds. Afterward, these ducks were allotted to 18 cages of 6 birds according to similar cage weight. Each dietary treatment had six replicate cages. The experimental diets were fed from 14 to 35 days of age. All ducks were given free access to water and feed and lighting was continuous. The temperature was kept at $33^{\circ} \mathrm{C}$ from 1 to 3 days of age and then it was reduced gradually to approximately $25^{\circ} \mathrm{C}$ until 14 days of age and was kept at approximately 16 to $22^{\circ} \mathrm{C}$ during the growing period from 14 to 35 days of age.

\section{Experimental Diets}

Experimental diets with low, medium, and high TDF levels were formulated and the feed composition of all these diets are provided in Table 1. All experimental diets were cold-pelleted at room temperature and the TDF levels of these diets also were analyzed according to the method of AOAC International (2007). The analyzed TDF levels of these 3 experimental diets were 12.4, 14.7 , and $16.2 \%$, respectively.

\section{Sample Collection}

At 35 days of age, the body weight (BW), average daily gain (ADG), average daily feed intake (ADFI), and feed to gain ratio $(\mathrm{F} / \mathrm{G})$ of ducks from each cage were measured. Feed intake 
TABLE 1 | Ingredient and composition of the experimental diets (\%, dry matter basis).

\begin{tabular}{|c|c|c|c|}
\hline \multirow[t]{2}{*}{ Item } & \multicolumn{3}{|c|}{ Content (\%) } \\
\hline & $\begin{array}{c}\text { Low TDF } \\
\text { diet }\end{array}$ & $\begin{array}{l}\text { Medium } \\
\text { TDF diet }\end{array}$ & $\begin{array}{c}\text { High TDF } \\
\text { diet }\end{array}$ \\
\hline Corn & 66.36 & 66.49 & 66.62 \\
\hline Soybean meal & 28.10 & 14.05 & 0.00 \\
\hline Isolated soybean protein & 0.00 & 6.00 & 12.00 \\
\hline Soybean dietary fiber & 0.00 & 6.35 & 12.70 \\
\hline Soy oil & 1.50 & 2.75 & 4.00 \\
\hline DL-Methionine & 0.14 & 0.175 & 0.21 \\
\hline L-Lysine-HCl & 0.00 & 0.035 & 0.07 \\
\hline L-Threonine & 0.00 & 0.065 & 0.13 \\
\hline L-Tryptophan & 0.00 & 0.035 & 0.07 \\
\hline Dicalcium phosphate & 1.50 & 1.55 & 1.60 \\
\hline Limestone & 1.10 & 1.20 & 1.30 \\
\hline Salt & 0.30 & 0.30 & 0.30 \\
\hline Vitamin-mineral premix ${ }^{a}$ & 1.00 & 1.00 & 1.00 \\
\hline \multicolumn{4}{|l|}{ Calculated nutrient levels } \\
\hline ME (Mcal/kg) & 2.92 & 2.91 & 2.90 \\
\hline Crude protein, \% & 17.67 & 17.685 & 17.70 \\
\hline Lysine, \% & 0.90 & 0.885 & 0.87 \\
\hline Methionine, \% & 0.41 & 0.41 & 0.41 \\
\hline Methionine + cystine, \% & 0.70 & 0.63 & 0.56 \\
\hline Tryptophan, \% & 0.22 & 0.215 & 0.21 \\
\hline Threonine, \% & 0.73 & 0.725 & 0.72 \\
\hline Calcium, \% & 0.80 & 0.80 & 0.80 \\
\hline Total phosphorus, \% & 0.61 & 0.58 & 0.55 \\
\hline Non-ohytate phosphorus, \% & 0.39 & 0.39 & 0.39 \\
\hline \multicolumn{4}{|l|}{ Analyzed value } \\
\hline TDF, \% & 12.4 & 14.7 & 16.2 \\
\hline
\end{tabular}

a Supplied per kilogram of total diet: Cu, $8 \mathrm{mg} ; \mathrm{Fe}, 60 \mathrm{mg}$; Zn, $60 \mathrm{mg} ; \mathrm{Mn}, 100 \mathrm{mg}$; Se, $0.3 \mathrm{mg}$; , $0.4 \mathrm{mg}$; choline chloride, 1,000 mg; vitamin A, 4,000 IU; vitamin D3, 2,000 U; vitamin E, $20 \mathrm{U}$; vitamin K3, $2 \mathrm{mg}$; thiamin, $2 \mathrm{mg}$; riboflavin, $10 \mathrm{mg}$; pyridoxine hydrochloride, $4 \mathrm{mg}$; cobalamin, $0.02 \mathrm{mg}$; calcium-D-pantothenate, $20 \mathrm{mg}$; nicotinic acid, $50 \mathrm{mg}$; folic acid, $1 \mathrm{mg}$; and biotin, $0.15 \mathrm{mg}$. TDF, total dietary fiber.

and F/G were all corrected for mortality. Afterward, two ducks were randomly selected from each cage and euthanized by $\mathrm{CO}_{2}$ inhalation. The two ceca of each selected ducks were collected. Cecal content was collected from one cecum for microbiota and SCFAs analysis, and mucosa was scraped by sterile blade after saline flush for gene expression analysis, and both of them were stored at $-80^{\circ} \mathrm{C}$. One centimeter in length of the other cecum was collected and fixed in 4\% paraformaldehyde solution for gut morphology analysis.

\section{Gut Morphology}

The samples of cecum fixed in $4 \%$ paraformaldehyde solution were embedded in paraffin, and then sectioned at $5 \mu \mathrm{m}$ and stained with hematoxylin and eosin using the standard procedures. The sections were pictured by Sony Alpha6000 APS camera. Then villus height (VH), crypt depth (CD), and muscle layer thickness (MLT) of cecum were analyzed by Image Pro-Plus
6.0 software (Media Cybernetics, Bethesda, MD, United States) at $100 \times$ magnification.

\section{SCFAs Analysis}

Cecal content was weighted approximately $0.5 \mathrm{~g}$ and diluted with $2 \mathrm{ml}$ ultrapure water, tempestuously commixed and centrifuged $\left(10,000 \mathrm{~g}, 15 \mathrm{~min}\right.$ at $\left.4^{\circ} \mathrm{C}\right)$ following $900 \mu \mathrm{l}$ supernatant being mixed with prepared $100 \mu \mathrm{l}$ ice-cold 25\% (w/v) metaphosphoric acid solution at $4^{\circ} \mathrm{C}$ for $4 \mathrm{~h}$ in a shaded environment. Then the mixture was centrifuged $\left(10,000 \mathrm{~g}, 15 \mathrm{~min}\right.$ at $\left.4^{\circ} \mathrm{C}\right)$ and the solution was filtered with $45 \mu \mathrm{m}$ nylon microporous membrane by syringe. The gas chromatography system measured concentrations of acetate, propionate, isobutyrate, butyrate, isovalerate, and valerate by DBFFAP column $(30 \mathrm{~m} \times 250 \mu \mathrm{m} \times 0.25 \mu \mathrm{m})$. The carrier gas was the $\mathrm{N}_{2}(12.5 \mathrm{Mpa}, 0.8 \mathrm{ml} / \mathrm{min})$. The temperature of FID detector was $280^{\circ} \mathrm{C}$ and that of column heated from 60 to $220^{\circ} \mathrm{C}$ at a rate of $20^{\circ} \mathrm{C} / \mathrm{min}$.

\section{Cecal Microbiota Analysis}

Total genomic DNA from cecal content samples was extracted by QIAamp DNA Stool Mini Kit (Qiagen, Hilden, Germany) according to the manufacturer's instructions. DNA concentration and integrality were detected by NanoDrop Spectrophotometer (Thermo Fisher Scientific, Wilmington, DE, United States) and agarose gel electrophoresis, respectively. DNA concentration of each cecal content was diluted to $10 \mathrm{ng} / \mu \mathrm{l}$ using double-distilled water. The V3-V4 region of $16 \mathrm{~S}$ rDNA was amplified using the following specific primers (338F:5'-ACTCCTACGGGAGGCAGCAG-3'; 806R: $5^{\prime}$-GGACTACHVGGGTWTCTAAT- $3^{\prime}$ ). Purified amplicons were pooled in equal amounts and paired-end sequenced $(2 \times 250 \mathrm{bp})$ on an Illumina MiSeq platform at Majorbio Bio-Pharm Technology Co., Ltd. (Shanghai, China).

\section{Gene Expression}

Total RNA from cecal mucous membrane was extracted by TRIzol reagent (Takara), according to the manufacturer's instruction book. Primer premier 6 was used to custom primers that are shown in Supplementary Table 1. The number of $1,000 \mathrm{ng}$ of total RNA was applied to synthesized cDNA using the PrimeScript RT Reagent Kit (Takara). ABI 7500 Real-time PCR Instrument implemented Real-time PCR (Applied Biosystems). PCR system was comprised of $1 \mu \mathrm{l}$ of five times diluted cDNA, $0.4 \mu \mathrm{l}$ each of $10 \mu \mathrm{M}$ forward and reverse primers, $5 \mu \mathrm{l}$ TB Green Premix Ex Taq II (Takara), $0.2 \mu$ l ROX Reference Dye II (Takara) and $3 \mu \mathrm{l}$ DNase Free $\mathrm{dH}_{2} \mathrm{O}$. The PCR amplification system was in two stages including $95^{\circ} \mathrm{C}$ for $30 \mathrm{~s}$, followed by 40 cycles both of $95^{\circ} \mathrm{C}$ for $5 \mathrm{~s}$ and $60^{\circ} \mathrm{C}$ for $34 \mathrm{~s}$. The specificity of the primers was examined by a melting curve analysis. The gene $\beta$-actin was selected as a reference gene, which was used to normalize the relative expression of genes of interest by the $2^{-\Delta \Delta C T}$ method.

\section{Statistical Analysis}

The data in a completely randomized design were analyzed using the one-way ANOVA procedure of SAS 9.4 software 
(SAS Institute, Inc.), with cage as the experimental unit for analyzing growth performance and each selected bird as the experimental unit for other parameters. $P<0.05$ was considered statistical significance. All data were expressed as means and pooled SEM.

For sequence processing, the raw reads were demultiplexed and quality-filtered by QIIME pipeline (version 1.17) (Edgar, 2010). Quality-filtered was performed to filter low-quality reads with average Phred scores lower than 20 and potential chimeric sequences were discarded using Uchime algorithm (Edgar et al., 2011). Then FLASH was used to merge reads (Magoc and Salzberg, 2011). The available sequences were clustered into operational taxonomic units (OTUs) according to $97 \%$ similarity. Taxonomy of OTUs were performed using the SILVA database. For $\alpha$-diversity analysis and $\beta$-diversity using principal coordinate analysis (PCoA) based on unweighted Unifrac distance, R package "vegan" and "ggplot2" were implemented to plot the results. The significant distinction of microbial communities was estimated by ANOSIM based on unweighted Unifrac distance with R package "vegan." The relative abundance of bacteria at family level was analyzed by one-way ANOVA among groups and Welch's $t$-test was applied to post hoc using R package "stats" and Python package "scipy." The different abundance of microbiota communities from phylum to genus was analyzed using the linear discriminant analysis (LDA) effect size (LEfSe) algorithm under the non-parametric factorial Kruskal-Wallis sum-rank test with $P<0.05$. LDA score $(>2)$ was to examine significant microbiota communities. Spearman correlation was used to analyze the relationship between microbial community taxa and variable factors including acetate, propionate, isobutyrate, butyrate, isovalerate, and valerate using $\mathrm{R}$ package "pheatmap" with $P<0.05$. Linear regression was devised to evaluate collinearity of variable factors using variance inflation factor (VIF) $(<10)$ with R package "tidyverse" and "caret."

\section{RESULTS}

\section{Growth Performance}

The effects of TDF on growth performance of White Pekin ducks are exhibited in Table 2. Compared with diets containing 12.4 and $14.7 \%$ TDF, the $16.2 \%$ TDF diet had distinctly ameliorated $(P<0.05)$ the BW of birds at 35 days. The ADG of birds significantly increased $(P<0.05)$ with increasing dietary fiber levels. The 16.2\% TDF treatments significantly lowered $(P<0.05)$ $\mathrm{F} / \mathrm{G}$ of birds compared with the experimental treatments containing 12.4 and $14.7 \%$ TDF.

\section{Cecal Morphology}

The parameters of sections of cecal morphology are shown in Table 3. The birds fed 14.7 and $16.2 \%$ TDF diets had significantly improved $(P<0.05) \mathrm{VH}$ compared with other birds fed $12.4 \%$ TDF diet. The 14.7 and $16.2 \%$ TDF treatments significantly lowered $(P<0.05)$ the CD of birds. The V/C and MLT in the cecum of birds were dramatically increased $(P<0.05)$ when birds fed $14.7 \%$ TDF diet.
TABLE 2 | Effects of total dietary fiber on growth performance of White Peking ducks on day 35.

\begin{tabular}{lccccc}
\hline Item & $\mathbf{1 2 . 4 \%}$ TDF & $\mathbf{1 4 . 7 \%}$ TDF & $\mathbf{1 6 . 2 \%}$ TDF & SEM & $\boldsymbol{P}$-value \\
\hline BW (g) & $2396^{\mathrm{b}}$ & $2461^{\mathrm{b}}$ & $2533^{\mathrm{a}}$ & 23.4 & $<0.01$ \\
$\mathrm{ADG}(\mathrm{g})$ & $87.0^{\mathrm{b}}$ & $90.0^{\mathrm{ab}}$ & $93.4^{\mathrm{a}}$ & 1.54 & $<0.01$ \\
$\mathrm{ADFI}(\mathrm{g})$ & 192 & 196 & 193 & 2.39 & 0.624 \\
F/G & $2.21^{\mathrm{b}}$ & $2.17^{\mathrm{b}}$ & $2.07^{\mathrm{a}}$ & 0.02 & $<0.01$ \\
\hline
\end{tabular}

a,b Means with different superscripts in the same row differ significantly ( $n=6$; $P<0.05)$. 12.4\% TDF, $12.4 \%$ total dietary fiber; $14.7 \%$ TDF, $14.7 \%$ total dietary fiber; $16.2 \%$ TDF, $16.2 \%$ total dietary fiber; BW, body weight; ADG, average daily gain; ADFl, average daily feed intake; F/G, the ratio of feed to gain.

TABLE 3 | Effects of total dietary fiber on cecal morphology of White Peking ducks on day 35.

\begin{tabular}{lccccc}
\hline Item & $\mathbf{1 2 . 4 \%}$ TDF & $\mathbf{1 4 . 7} \%$ TDF & $\mathbf{1 6 . 2} \%$ TDF & SEM & $P$-value \\
\hline $\mathrm{VH}(\mu \mathrm{m})$ & $487^{\mathrm{b}}$ & $622^{\mathrm{a}}$ & $646^{\mathrm{a}}$ & 16.9 & $<0.01$ \\
$\mathrm{CD}(\mu \mathrm{m})$ & $123^{\mathrm{b}}$ & $100^{\mathrm{a}}$ & $105^{\mathrm{a}}$ & 3.34 & $<0.01$ \\
$\mathrm{~V} / \mathrm{C}$ & $3.99^{\mathrm{b}}$ & $6.26^{\mathrm{a}}$ & $6.21^{\mathrm{a}}$ & 0.230 & $<0.01$ \\
$\mathrm{MLT}(\mu \mathrm{m})$ & $281^{\mathrm{c}}$ & $359^{\mathrm{a}}$ & $330^{\mathrm{b}}$ & 8.99 & $<0.01$ \\
\hline
\end{tabular}

$\overline{a-c}$ Means with different superscripts in the same row differ significantly ( $n=6$; $P<0.05)$. 12.4\% TDF, $12.4 \%$ total dietary fiber; $14.7 \%$ TDF, $14.7 \%$ total dietary fiber; 16.2\% TDF, 16.2\% total dietary fiber; VH, villus height; $C D$, crypt depth; V/C, the ratio of $\mathrm{VH}$ to $\mathrm{CD}$; MLT, muscle layer thickness.

\section{SCFAs Profiling}

The concentrations of SCFAs were measured and are shown in Figure 1. The concentration of propionate was higher $(P<0.05)$ in the cecum of ducks fed $12.4 \%$ TDF diet compared with other experimental diets. Butyrate concentration was conspicuous $(P<0.05)$ in the diets containing $14.7 \%$ TDF compared with 12.4 and $16.2 \%$ TDF diets. The concentrations of isobutyrate, isovalerate, and valerate were significantly increased $(P<0.05)$ in the $16.2 \%$ TDF diet relative to 12.4 and $16.2 \%$ TDF diets.

\section{Cecal Microbial Analysis}

After quality-filtered and merge, the average of 22,036 available sequences of each sample was generated, and 1,010 OTUs were obtained. Rarefaction curves analysis based on sobs index showed sample sequencing depths were sufficient to cover almost all microbes in the samples (Figure 2). Compared with $12.4 \%$ TDF groups, the $\alpha$-diversity estimators (Figure 3 ) of richness including ace and chaol were significantly increased $(P<0.05)$ in 14.7 and $16.2 \%$ TDF groups, and that of diversity assessed by Shannon were conspicuously raised $(P<0.05)$. Microbial profile was clustered using PCoA (Figure 4) based on unweighted Unifrac distance, by which cecal microbial communities of ducks fed three levels of dietary fiber diet were distributed three detached clusters. ANOSIM method based on unweighted Unifrac distance ulteriorly illustrated that microbial communities was the significant distinction in different content of dietary fiber groups $\left(R^{2}=0.336, P<0.05\right)$ indicating that increasing dietary fiber exerted an effect on microbial composition.

In order to analyze microbial composition, phyla, family, and genus were selected as taxonomic levels. Firmicutes, Bacteroidota, Actinobacteriota, and Desulfobacterota were the 


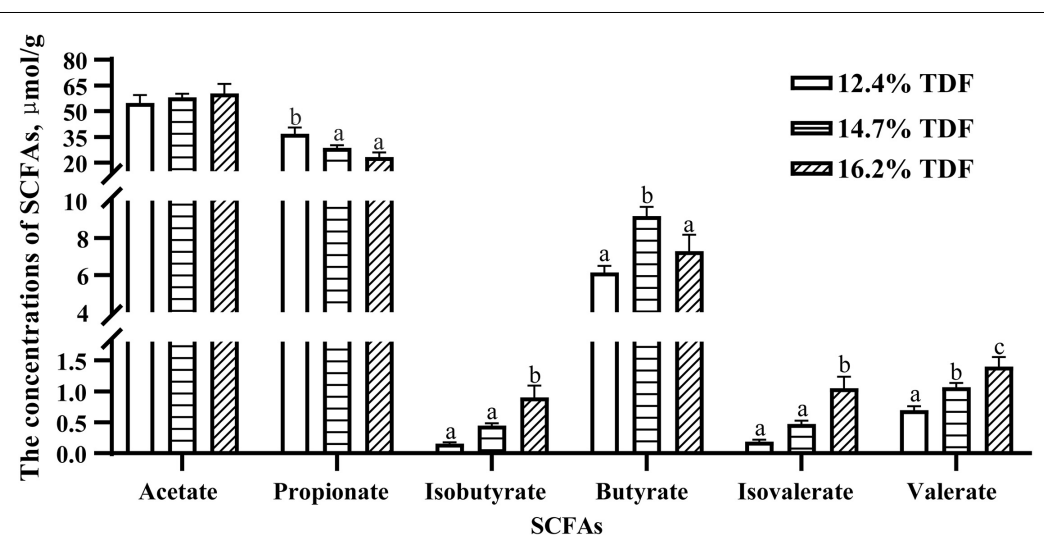

FIGURE 1 | Effects of total dietary fiber on the concentrations of cecal SCFAs of White Peking ducks on day $35 .{ }^{a-c}$ Means with different superscripts in the same index differ significantly $(n=12, P<0.05)$. 12.4\% TDF, $12.4 \%$ total dietary fiber; $14.7 \%$ TDF, $14.7 \%$ total dietary fiber; $16.2 \%$ TDF, $16.2 \%$ total dietary fiber; SCFAs, short-chain fatty acids.

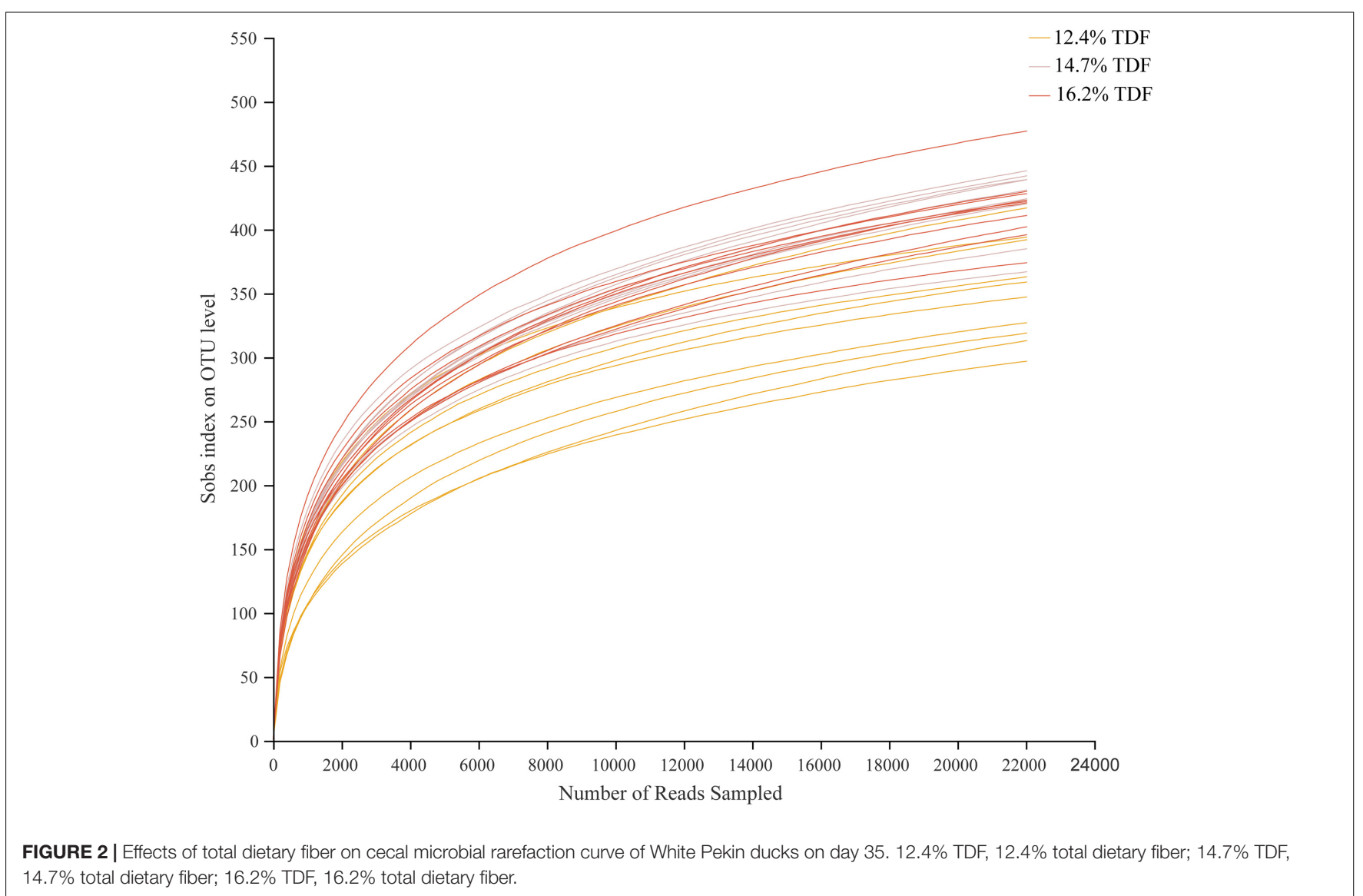

main phyla $(>1 \%)$ in the cecum of duck, and Firmicutes and Bacteroidota were the dominant phyla while the relative abundance of Firmicutes was higher than that of Bacteroidota in all groups (Figure 5A). At genus level, the heatmap (Figure 5B) of top 30 most relative abundance genus showed cecal microbial communities of duck fed $12.4 \%$ and $14.7 \%$ TDF diets formed a common cluster while that of duck fed $16.2 \%$ TDF diet structured a separate cluster indicating microbial composition was similar between $12.4 \%$ TDF group and $14.7 \%$ TDF group. In addition, cecal microbiota were mainly clustered into two groups according to the relative abundance (Figure 5B), which one of them had higher relative abundance including Bacteroides and Megamonas. The duck's cecal microbial communities of three groups was predominated by sequences representative of Bacteroidaceae, Ruminococcaceae and Lachnospiraceae at family level (Figure 5C). Compared with $12.4 \%$ TDF group, 

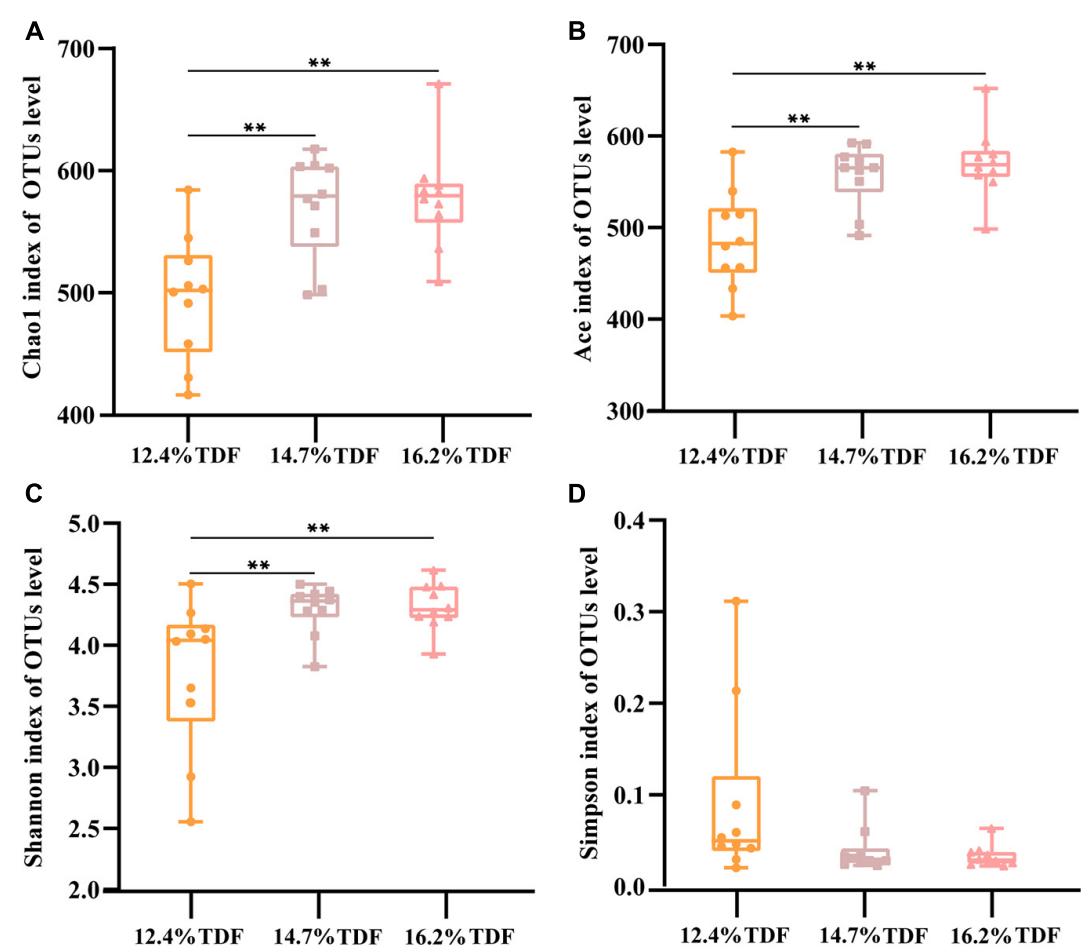

FIGURE 3 | Effects of total dietary fiber on cecal microbial diversity of White Peking ducks on day 35 . Significant difference was recorded by $0.01<P \leq 0.05^{*}$, $0.001<P \leq 0.01 * *, P \leq 0.001 * * * .12 .4 \%$ TDF, $12.4 \%$ total dietary fiber; $14.7 \%$ TDF, $14.7 \%$ total dietary fiber; $16.2 \%$ TDF, $16.2 \%$ total dietary fiber.

the relative abundance of Lachnospiraceae, Oscillospiraceae and Coriobacteriacea at family level was distinctly ameliorated $(P<0.05)$ after ducks fed $16.2 \%$ TDF diet. Besides, the relative abundance of Butyricicoccacea was in a significant rise $(P<0.05)$ in the $14.7 \%$ TDF group at family level (Figure 5D). Bacterodies and Megamonas were prominent genus in the cecum of duck, but the relative of Megamonas was lowered with increasing TDF level (Figure 5E). Firmicutes to Bacteroidota ratio was raised in the higher dietary fiber diet (Figure 5F).

Furthermore, LEfSe analysis (Figure 6) was explored to identify significant taxa in phylotypes. In the aggregate, 14 genera were detected with LDA threshold $>2$. Ducks that were fed $16.2 \%$ TDF diet had significantly enriched $(P<0.05)$ Bacilli relative abundance at class level. The relative abundance of Anaerovoracaceae, Oscillospiraceae, Christensenellaceae, Enterococcaceae, Norank_o_clostridia_UCG_014, and unclassified_o_Lactobacillales at at family level was increased $(P<0.05)$ compared with ducks fed lower TDF diets. Moreover, the genus Turicibacter and Romboutsia were biomarkers in the $14.7 \%$ TDF group. Besides, the cecal microbial community of ducks was characterized $(P<0.05)$ by Prevotellaceae_Ga6A1_group as a biomarker when ducks were fed $12.4 \%$ TDF diet.

\section{Correlations Between SCFAs and Microbiota}

To analyze microbiota associated with SCFAs as its metabolites, spearman analysis (Figure 7) was taken to evaluate the

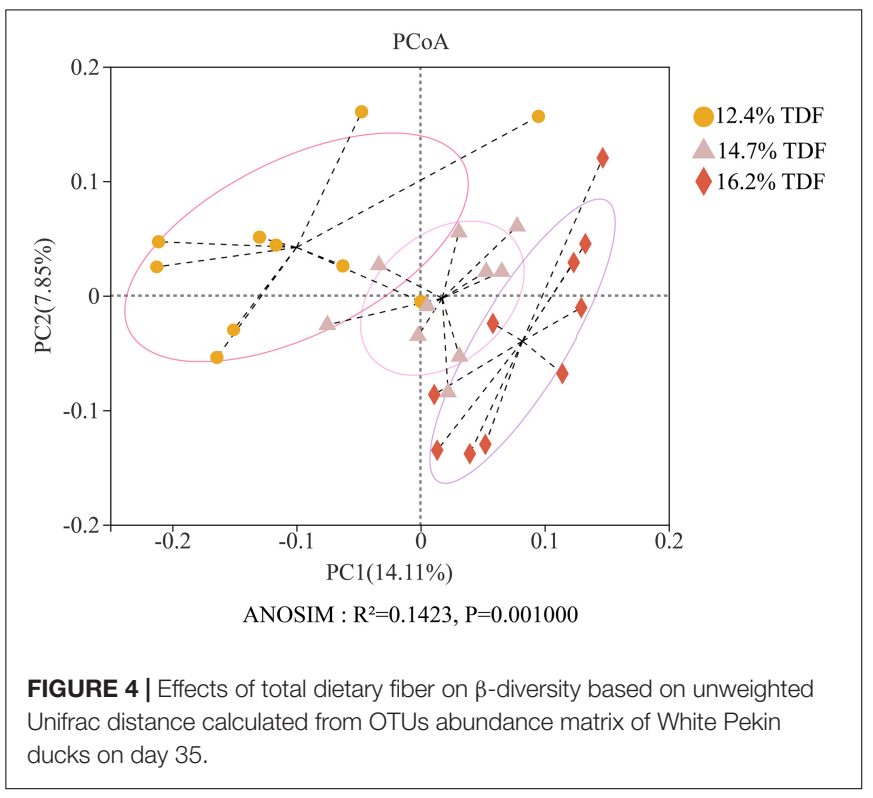

associations between SCFAs with VIF $<10$ in the cecum of ducks and top 20 most abundant genus. The concentrations of acetate and propionate were positively associated with Ruminococcus_torques_group $(P<0.05)$ and Bacteroides $(P<0.05)$ respectively, whereas propionate was negatively linked with Streptococcus, Enterococcus, and Faecalibacterium 


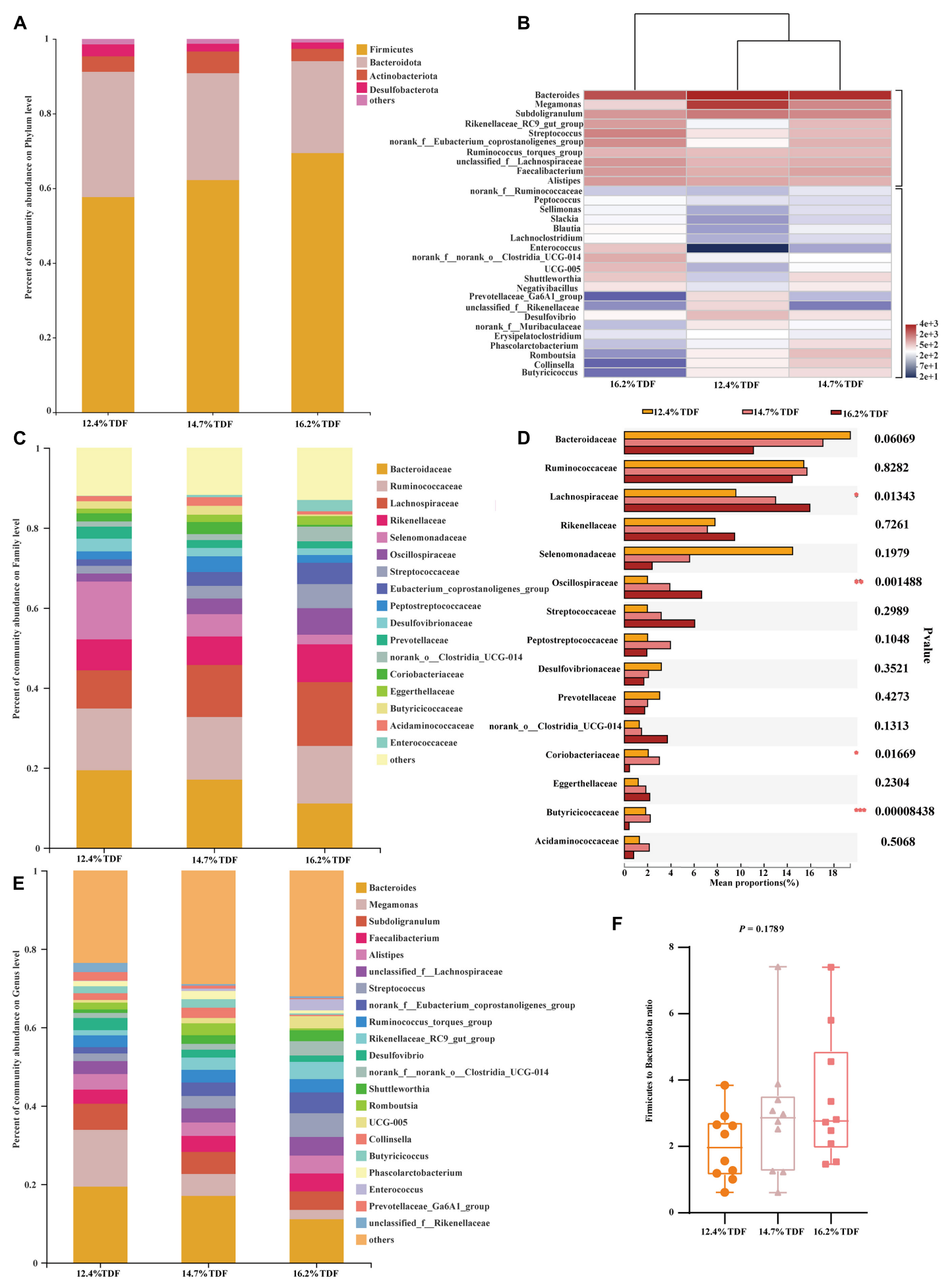

FIGURE 5 | Effects of total dietary fiber on composition of cecal microbiota and differential species identified at phylum, family and genus level of White Pekin ducks on day 35. (A,C,E) were microbiota composition at phylum, family, and genus level, respectively; (B) was the heatmap of top 30 relative abundance genus of microbiota communities; (D) was differential bacteria at family level and $\mathbf{( F )}$ was the ratio of the abundance of Firmicutes to Bacteroidetes at phylum level. Significant difference was recorded by $0.01<P \leq 0.05^{\star}, 0.001<P \leq 0.01^{\star \star}, P \leq 0.001^{* \star}$. 


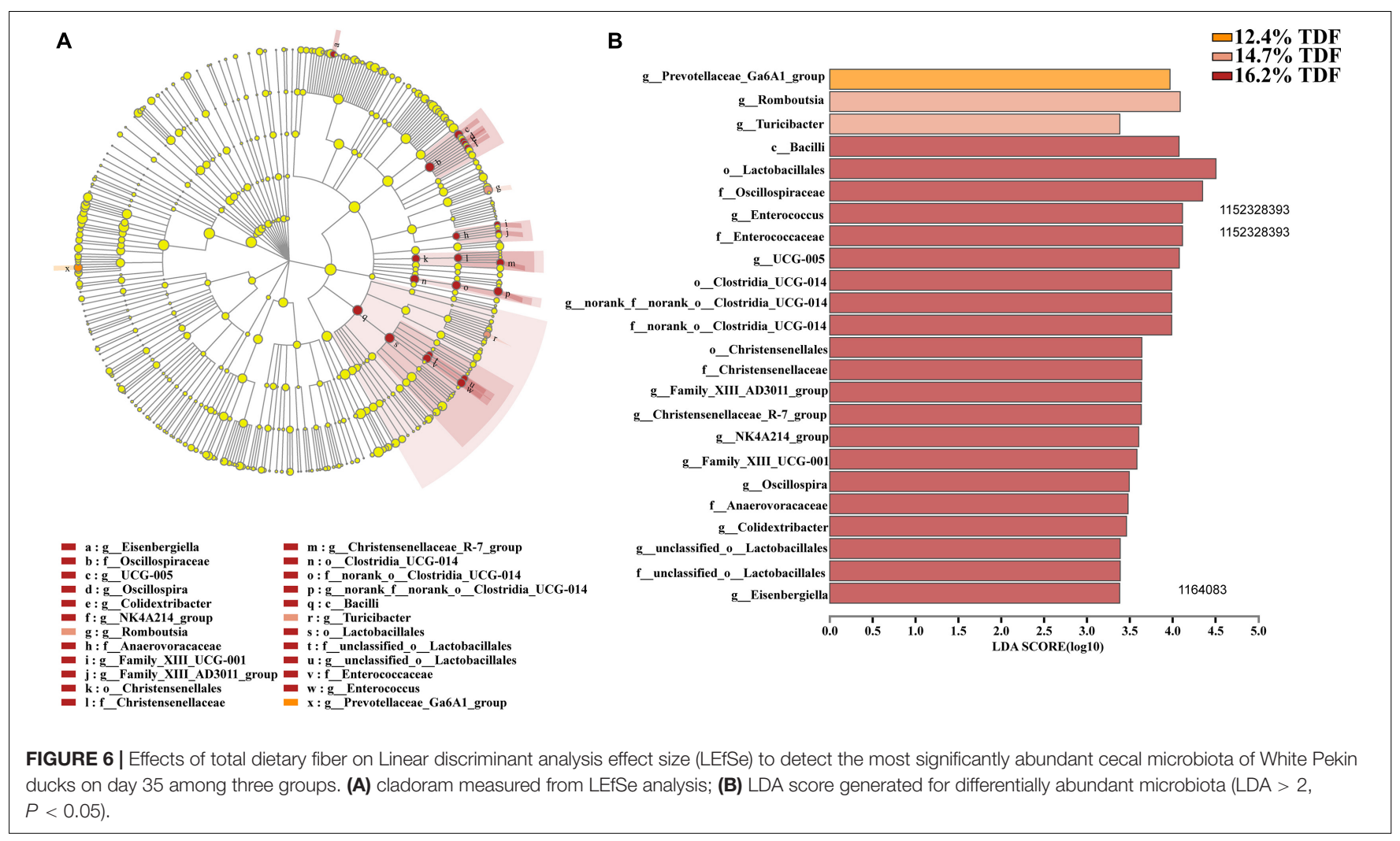

$(P<0.05)$. Isobutyrate and valerate had a significantly positive correlation with UCG-005 and Enterococcus $(P<0.05)$ while Romboutsia and Collinsella presented a negative correlation with isobutyrate $(P<0.05)$.

\section{Gene Expression}

The effects of TDF on cecum barrier function were investigated and the relative mRNA expression of intestinal barrier genes including Zonula occludens-1 (ZO-1), Mucin-2 (Muc2), Occludin, and Claudin-1 was shown in Figure 8. The expression of Claudin1 gene was distinctly raised $(P<0.05)$ in the cecum of ducks supplemented with 14.7 and $16.2 \%$ TDF diets. When compared with the $12.7 \% \mathrm{TDF}$, the $14.7 \%$ TDF diets significantly upregulated $(P<0.05)$ the expression of $M u c 2$ and $Z O-1$ genes.

\section{DISCUSSION}

In our study, increasing TDF levels could promote duck growth by increasing weight gain, and it was accompanied with the markedly improvement in cecal morphology and barrier genes expression at this instance, which indicated the growth promotion was closely related to intestinal mucosal integrity. The present study showed that high dietary TDF could enhance the cecal mucosal integrity of ducks by increasing VH and MLT and lowering $\mathrm{CD}$, and it was accompanied by increasing barrier gene expression of ZO-1, Claudin-1, and Muc2 at high dietary TDF level. Our results were partly supported by Han et al. (2017), who observed that the growth promotion of starter Pekin ducks caused by high crude fiber was accompanied with increasing gizzard weight and improved jejunal morphology. Furthermore, when Pekin ducks were fed resistant starch-supplemented diets, the increasing MLT, VH, and V/C of ileum or cecum was followed by the markedly increasing genes expression of ZO1, Claudin-1, and Muc2 in these tissues (Qin et al., 2019, 2020). Tight junction proteins are contributed to the intercellular junctional complexes between intestinal epithelial cells, which sealed the paracellular space between these cells and regulated the permeability of the intestinal barrier (Ulluwishewa et al., 2011). Mucins are synthesized and secreted from goblet cells, and they are the major component of the mucus layer (Montagne et al., 2004; Johansson, 2014). All these proteins are contributed to intestinal mucosal integrity and played crucial roles in gastrointestinal protection. Therefore, the duck cecal histological development regulated by high dietary TDF may be mediated by increasing gene expression of tight junction protein and mucins at this instance.

The diversity in intestinal microbiota based on the amount of OTUs was altered by the levels of dietary fiber. There was conspicuously different in $\alpha$-diversity among the three levels of dietary fiber groups. The estimators of ace and chaol ameliorated with increasing dietary fiber levels, indicating that dietary fiber could positively modulate species richness. Shannon index value was higher in the $16.2 \%$ TDF group, in which species diversity was more blooming. In line with previous study, Qin et al. (2020) reported dietary fiber had beneficial effects on diversity of cecal microbial communities of ducks. With the abundant bacterial diversity in the poultry hindgut, competitive inhibition 


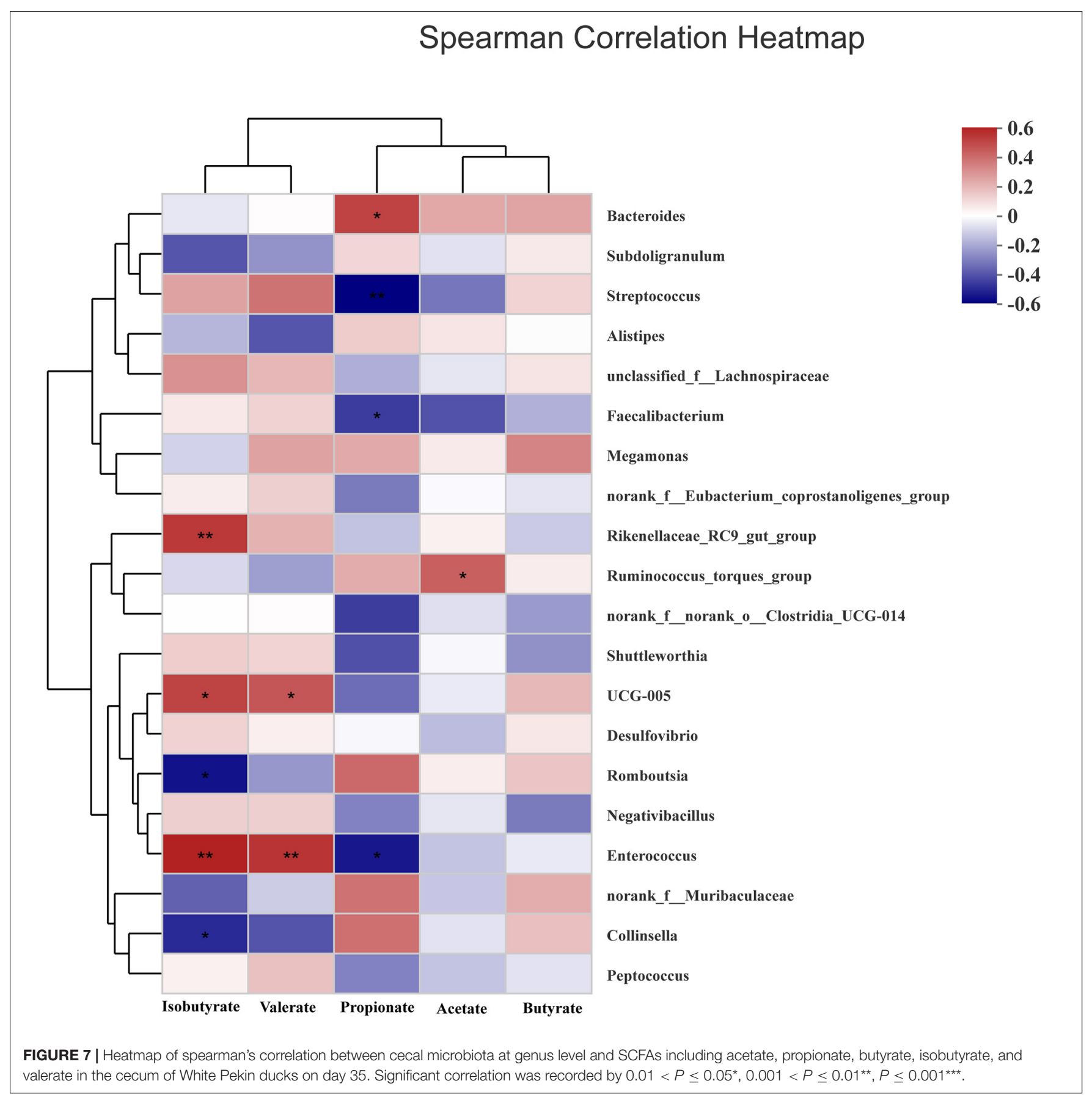

on pathogenic bacteria could be magnified (Zheng et al., 2019). Community structures of all groups were compared by PCoA analysis. The PCoA plot revealed cecal microbial communities were distributed in three detached clusters, which showed microbial composition was distinct among three levels of dietary fiber. Further, ANOSIM analysis confirmed the separate clusters in different content of dietary fiber groups were significantly dissimilar $\left(R^{2}=0.336, P<0.05\right)$. Consequently, it is reasonable to modulate microbial profile in the hindgut of ducks with dietary fiber diet.
Dietary fiber could alter microbial flora to present its function of healthy facilitation. Microbiota in the poultry gut was regarded as a barrier to defend against disease by eliminating pathogens and enhancing the immune system (Oakley et al., 2014). In the present study, Firmicutes and Bacteroidota were still the dominant phyla and Firmicutes had higher relative abundance, though ducks were fed different levels of dietary fiber diet, which was in line with the study of Wei et al. (2013). The improvement in the relative abundance of Firmicutes could bring benefits to normal colonization of microbial communities. 


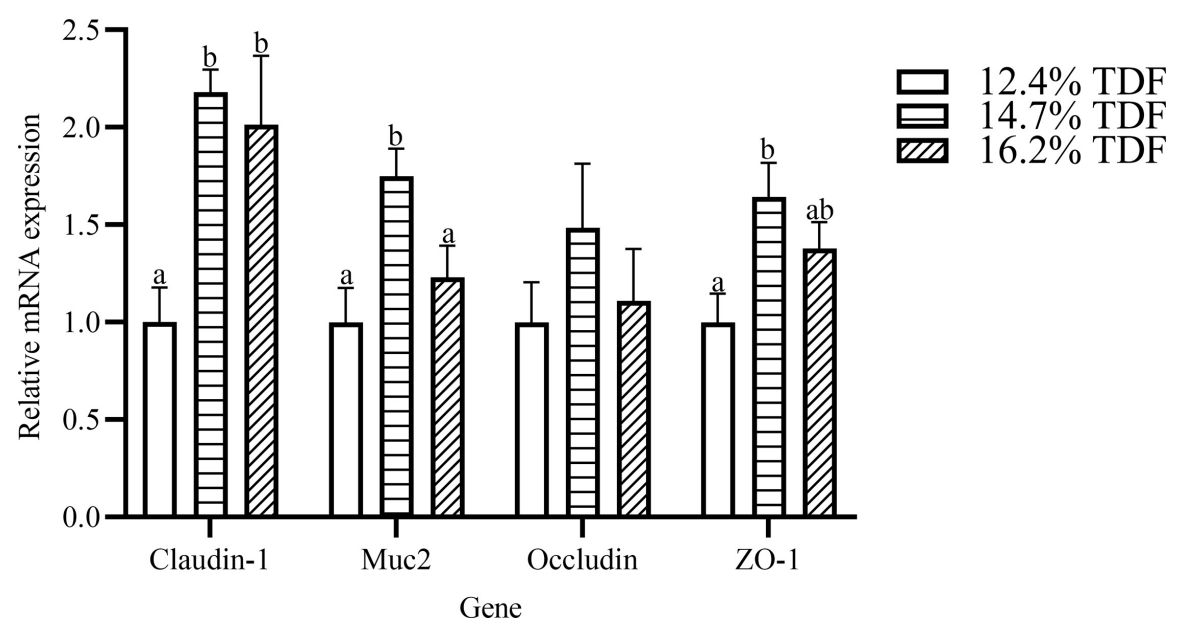

FIGURE 8 | Effects of total dietary fiber on the relative mRNA expression of cecal barrier function genes of White Pekin Ducks on day $35 .{ }^{a, b}$ Means with different superscripts in the same index differ significantly ( $n=6, P<0.05) .12 .4 \%$ TDF, $12.4 \%$ total dietary fiber; $14.7 \%$ TDF, $14.7 \%$ total dietary fiber; $16.2 \%$ TDF, $16.2 \%$ total dietary fiber; Muc2, Mucin-2; ZO-1, Zonula occludens-1.

Mulder et al. (2009) indicated that diet supplemented with dietary fiber had a positive role in minimizing pathogens. In the current study, higher Firmicutes to Bacteroidota ratio was facilitated by dietary fiber. The raised abundance of family Oscillospiraceae and Lachnospiraceae at expense of abundance of family Bacteroidaceae and Selenomondaceae contributed to higher Firmicutes to Bacteroidota ratio. Molist et al. (2012) reported dietary fiber promoted higher Firmicutes to Bacteroidota ratio in colon of piglets resulting in the reduced pathogenic infection chance. Oscillospiraceae and Lachnospiraceae known as butyrate-producing bacteria fermented dietary fiber into butyrate to limit pathogenic bacteria colonization (Guilloteau et al., 2010; Meehan and Beiko, 2014). Moreover, the positive correlation between genus Bacteroides of family Bacteroidaceae and pro-inflammatory cytokines including IL- 8 and TNF- $\alpha$ was reported by previous study (Wang et al., 2019). Therefore, the counterbalance of intestinal microbiota modulated by dietary fiber could be a biomarker to estimate intestinal lumen function of duck.

Admittedly, the changes in the relative abundance of microbiota in the gut ecosystem are pronouncedly linked with its metabolites. It was observed that dietary fiber as a substrate for microbial fermentation could be catabolized into SCFAs (Walugembe et al., 2015). It was established that the sequences representative of Firmicutes and Bacteroidota were major executors in the pathway of SCFAs metabolism, in which Firmicutes catabolized dietary fiber into propionate and butyrate while Bacteroidota specialized in producing propionate (Pandit et al., 2018). The relative abundance of Lachnospiraceae and Oscillopiraceae, butyrate producers (Biddle et al., 2013; Gophna et al., 2017), affiliating to Firmicutes significantly increased when ducks were fed higher levels of dietary fiber diet. Walugembe et al. (2015) reported that the relative abundance of butyrate producers like Ruminococcaceae was increased in the cecum of laying-hen chicks in the high dietary fiber group. Chen et al. (2020) also observed that dietary fiber could not only ameliorate the concentration of butyrate, but elevate the relative abundance of microbiota producing butyrate in the cecum of weaning piglets. In agreement with our study, the relative abundance of butyric-producing bacteria including Lachnospiraceae and Oscillopiraceae distinctly increased, by which Firmicutes abundance presented an ascent, and butyrate as its production also subsequently changed with an increase in TDF. Intriguingly, the expectedly augmented value of butyrate concentration as $14.7 \%$ TDF diet was lessening after supplying $16.2 \%$ TDF diet, which might be associated with the decline in the relative abundance of Butyricicoccus in the $16.2 \%$ TDF group. Since Butyricicoccus, pertaining to family Butyricicoccaceae, is a branch of producing-butyric bacteria colonizing the hindgut (Nava and Stappenbeck, 2011; Zvanych et al., 2014). However, the concentration of propionate was lowered with an increase in TDF that was in contrast to the findings of Qin et al. (2020), who observed that dietary fiber could ameliorate the concentration of propionate in the cecum. The difference could be partially explained by the lower abundance of Megamonas in the higher levels of dietary fiber groups in the present study given that Megamonas favored the pathway of propionate fermentation (Sergeant et al., 2014). Besides, Bacteroides presented a positive correlation with propionate from our observation corroborating the conclusion that Bacteroides were actively involved in the propionate fermentation pathway (Sergeant et al., 2014). The results illustrated microbial ecosystem plays a particular role in dietary fiber degradation and SCFAs production. Meanwhile, increasing dietary fiber has a distinct advantage in modulating microbial structure in the cecum of ducks.

Short-chain fatty acids were the major metabolites of dietary fiber fermentation by microbiota in the cecum of duck. Butyrate, one of the fermentable products, not only played a key role in energy salvage but improved the function of the intestinal barrier (Peng et al., 2009). Muc2 was a glycoprotein layer on the 
epithelial cell to structure mucus barrier, which was modulated by $M u c 2$ gene, and butyrate is beneficial to up-regulate the expression of Muc2 gene (Smirnov et al., 2005). In the present study, the expression of $M u c 2$ gene was conspicuously raised in the diet containing $14.7 \%$ TDF with a higher concentration of butyrate. In line with our study, Wellington et al. (2020) reported that high dietary fiber treatment elevated butyrate concentration and Muc2 gene expression in the cecum. Trachsel et al. (2018) revealed that dietary fiber diet containing 5\% resistant potato starch ameliorated concentration of butyrate and stimulated the expression of $M u c 2$ gene in the cecum. Besides, tight junctions could decrease paracellular permeability to limit pathogens and antigens (Groschwitz and Hogan, 2009). The tight junctions were mainly comprised of claudins, occludins, and zonula occludins, which were positively stimulated by butyrate (Kim et al., 2012). The expression of Claudin-1 and ZO-1 genes in the cecum increased with higher concentration of butyrate after ducks being fed dietary fiber diets containing 14.7 and 16.2\% TDF. The results were in accordance with the study of Chen et al. (2013), who revealed that dietary fiber could elevate butyrate concentration and the expression of Claudin-1 and ZO-1 genes in the ileum of piglets. Therefore, combined with the improvement of cecal morphology and barrier genes expressing at high dietary TDF, it could be speculated that increasing dietary TDF could produce more butyrate by altering intestinal microbiota to stimulate intestinal development in ducks.

\section{CONCLUSION}

In a nutshell, increasing TDF levels could bring benefits to growth performance and intestinal health in meat ducks. Important biomarkers like elevated cecal histomorphology parameters, changed microbiota communities, enhanced SCFAs concentrations, and up-regulated expression of barrier-related genes were observed. Meanwhile, these results powerfully revealed that changes of cecal microbiota communities might conduce to the improvement of butyrate concentration, which subsequently exerted beneficial effects on the intestinal

\section{REFERENCES}

AOAC International (2007). Official Methods of Analysis of AOAC International. Gaithersburg, MD: Oxford University Press.

Biddle, A., Stewart, L., Blanchard, J., and Leschine, S. (2013). Untangling the genetic basis of fibrolytic specialization by Lachnospiraceae and ruminococcaceae in diverse gut communities. Diversity 3, 627-640. doi: 10.3390/d5030627

Chen, T., Chen, D., Tian, G., Zheng, P., Mao, X., Yu, J., et al. (2020). Effects of soluble and insoluble dietary fiber supplementation on growth performance, nutrient digestibility, intestinal microbe and barrier function in weaning piglet. Anim. Feed Sci. Technol. 260:114335. doi: 10.1016/j.anifeedsci.2019.114335

Chen, H., Mao, X., He, J., Yu, B., Huang, Z., Yu, J., et al. (2013). Dietary fibre affects intestinal mucosal barrier function and regulates intestinal bacteria in weaning piglets. Br. J. Nutr. 10, 1837-1848. doi: 10.1017/S0007114513001293

Du, X., Xiang, Y., Lou, F., Tu, P., Zhang, X., Hu, X., et al. (2020). Microbial community and short-chain fatty acid mapping in the intestinal tract of quail. Animals 10:1006. doi: 10.3390/ani10061006

Edgar, R. C. (2010). Search and clustering orders of magnitude faster than BLAST. Bioinformatics 19, 2460-2461. doi: 10.1093/bioinformatics/btq461 barrier function, facilitating the interaction between intestinal mucosa and microbiota.

\section{DATA AVAILABILITY STATEMENT}

The datasets presented in this study can be found in online repositories. The names of the repository/repositories and accession number(s) can be found below: https://www.ncbi.nlm. nih.gov/, PRJNA731120.

\section{ETHICS STATEMENT}

The animal study was reviewed and approved by animal care and use committee of Institute of Animal Sciences of Chinese Academy of Agricultural Sciences.

\section{AUTHOR CONTRIBUTIONS}

$\mathrm{YH}$ implemented the study and wrote the manuscript. YH, ZS, ZJ, YW, BZ, and JT assisted in conducting the experiment and collecting samples. $\mathrm{SH}$ and MX designed the experiment. MX revised the manuscript. All authors contributed to the study and supported the submitted version.

\section{FUNDING}

This study was supported by China Agriculture Research System of MOF and MARA (CARS-42).

\section{SUPPLEMENTARY MATERIAL}

The Supplementary Material for this article can be found online at: https://www.frontiersin.org/articles/10.3389/fmicb. 2021.727200/full\#supplementary-material

Edgar, R. C., Haas, B. J., Clemente, J. C., Quince, C., and Knight, R. (2011). UCHIME improves sensitivity and speed of chimera detection. Bioinformatics 16, 2194-2200. doi: 10.1093/bioinformatics/btr381

Gophna, U., Konikoff, T., and Nielsen, H. B. (2017). Oscillospira and related bacteria-From metagenomic species to metabolic features. Environ. Microbiol. 19, 835-841. doi: 10.1111/1462-2920.13658

Groschwitz, K. R., and Hogan, S. P. (2009). Intestinal barrier function: molecular regulation and disease pathogenesis. J. Allergy Clin. Immunol. 124, 3-20. doi: 10.1016/j.jaci.2009.05.038

Guilloteau, P., Martin, L., Eeckhaut, V., Ducatelle, R., Zabielski, R., and Van Immerseel, F. (2010). From the gut to the peripheral tissues: the multiple effects of butyrate. Nutr. Res. Rev. 23, 366-384. doi: 10.1017/S09544224100 00247

Han, H. Y., Zhang, K. Y., Ding, X. M., Bai, S. P., Luo, Y. H., Wang, J. P., et al. (2017). Effect of dietary fiber levels on performance, gizzard development, intestinal morphology, and nutrient utilization in meat ducks from 1 to 21 days of age. Poult. Sci. 12, 4333-4341. doi: 10.3382/ps/pex268

Johansson, M. E. (2014). Mucus layers in inflammatory bowel disease. Inflamm. Bowel Dis. 11, 2124-2131. doi: 10.1097/MIB.0000000000000117 
Kheravii, S. K., Morgan, N. K., Swick, R. A., Choct, M., and Wu, S. B. (2018). Roles of dietary fibre and ingredient particle size in broiler nutrition. Worlds Poult. Sci. J. 74, 301-316. doi: 10.1017/s0043933918000259

Kim, J. C., Hansen, C. F., Mullan, B. P., and Pluske, J. R. (2012). Nutrition and pathology of weaner pigs: nutritional strategies to support barrier function in the gastrointestinal tract. Anim. Feed Sci. Technol. 20, 3-16. doi: 10.1016/j. anifeedsci.2011.12.022

Magoc, T., and Salzberg, S. L. (2011). FLASH: fast length adjustment of short reads to improve genome assemblies. Bioinformatics 21, 2957-2963. doi: 10.1093/ bioinformatics/btr507

Meehan, C. J., and Beiko, R. G. (2014). A phylogenomic view of ecological specialization in the Lachnospiraceae, a family of digestive tract-associated bacteria. Genome Biol. Evol. 3, 703-713. doi: 10.1093/gbe/evu050

Molist, F., Manzanilla, E. G., Perez, J. F., and Nyachoti, C. M. (2012). Coarse, but not finely ground, dietary fibre increases intestinal firmicutes:bacteroidetes ratio and reduces diarrhoea induced by experimental infection in piglets. Br. J. Nutr. 1, 9-15. doi: 10.1017/S0007114511005216

Montagne, L., Piel, C., and Lallès, J. P. (2004). Effect of diet on mucin kinetics and composition: nutrition and health implications. Nutr. Rev. 3, 105-114. doi: 10.1301/nr.2004.mar.105-114

Mulder, I. E., Schmidt, B., Stokes, C. R., Lewis, M., Bailey, M., Aminov, R. I., et al. (2009). Environmentally-acquired bacteria influence microbial diversity and natural innate immune responses at gut surfaces. BMC Biol. 7:79. doi: 10.1186/1741-7007-7-79

Nava, G. M., and Stappenbeck, T. S. (2011). Diversity of the autochthonous colonic microbiota. Gut Microbes 2, 99-104. doi: 10.4161/gmic.2.2.15416

Oakley, B. B., Lillehoj, H. S., Kogut, M. H., Kim, W. K., Maurer, J. J., Pedroso, A., et al. (2014). The chicken gastrointestinal microbiome. FEMS Microbiol. Lett. 2, 100-112. doi: 10.1111/1574-6968.12608

Pandit, R. J., Hinsu, A. T., Patel, N. V., Koringa, P. G., Jakhesara, S. J., Thakkar, J. R., et al. (2018). Microbial diversity and community composition of caecal microbiota in commercial and indigenous Indian chickens determined using 16s rDNA amplicon sequencing. Microbiome 6:115. doi: 10.1186/s40168-0180501-9

Peng, L. Y., Li, Z. R., Green, R. S., Holzman, I. R., and Lin, J. (2009). Butyrate enhances the intestinal barrier by facilitating tight junction assembly via activation of AMP-activated protein kinase in Caco-2 cell monolayers. J. Nutr. 9, 1619-1625. doi: 10.3945/jn.109.104638

Pu, G., Li, P., Du, T., Niu, Q., Fan, L., Wang, H., et al. (2020). Adding appropriate fiber in diet increases diversity and metabolic capacity of distal gut microbiota without altering fiber digestibility and growth rate of finishing pig. Front. Microbiol. 11:533. doi: 10.3389/fmicb.2020.00533

Qin, S. M., Zhang, K. Y., Ding, X. M., Bai, S. P., Wang, J. P., and Zeng, Q. F. (2019). Effect of dietary graded resistant potato starch levels on growth performance, plasma cytokines concentration, and intestinal health in meat ducks. Poult. Sci. 98, 3523-3532. doi: 10.3382/ps/pez186

Qin, S., Han, H., Zhang, K., Ding, X., Bai, S., Wang, J., et al. (2018). Dietary fibre alleviates hepatic fat deposition via inhibiting lipogenic gene expression in meat ducks. J. Anim. Physiol. Anim. Nutr. 2, e736-e745. doi: 10.1111/jpn. 12828

Qin, S., Zhang, K., Applegate, T. J., Ding, X., Bai, S., Luo, Y., et al. (2020). Dietary administration of resistant starch improved caecal barrier function by enhancing intestinal morphology and modulating microbiota composition in meat duck. Br. J. Nutr. 123, 172-181. doi: 10.1017/S00071145190 02319

Sergeant, M. J., Constantinidou, C., Cogan, T. A., Bedford, M. R., Penn, C. W., and Pallen, M. J. (2014). Extensive microbial and functional diversity within the chicken cecal microbiome. PLoS One 9:e91941. doi: 10.1371/journal.pone. 0091941

Singh, A. K., and Kim, W. K. (2021). Effects of dietary fiber on nutrients utilization and gut health of poultry: a review of challenges and opportunities. Animals 11:181. doi: 10.3390/ani11010181
Smirnov, A., Perez, R., Amit-Romach, E., Sklan, D., and Uni, Z. (2005). Mucin dynamics and microbial populations in chicken small intestine are changed by dietary probiotic and antibiotic growth promoter supplementation. J. Nutr. 135, 187-192.

Tang, D., Li, Z., Mahmood, T., Liu, D., Hu, Y., and Guo, Y. (2020). The association between microbial community and ileal gene expression on intestinal wall thickness alterations in chickens. Poult. Sci. 99, 1847-1861. doi: 10.1016/j.psj. 2019.10.029

Tejeda, J. O., and Kim, W. K. (2021). Role of dietary fiber in poultry nutrition. Animals 11:461. doi: 10.3390/ani11020461

Tejeda, O. J., and Kim, W. K. (2020). The effects of cellulose and soybean hulls as sources of dietary fiber on the growth performance, organ growth, gut histomorphology, and nutrient digestibility of broiler chickens. Poult. Sci. 99, 6828-6836. doi: 10.1016/j.psj.2020.08.081

Trachsel, J., Briggs, C., Gabler, N. K., Allen, H. K., and Loving, C. L. (2018). Resistant potato starch fuels beneficial host-microbe interactions in the gut. bioRxiv 389007 [Preprint]. doi: 10.1101/389007

Ulluwishewa, D., Anderson, R. C., McNabb, W. C., Moughan, P. J., Wells, J. M. and Roy, N. C. (2011). Regulation of tight junction permeability by intestinal bacteria and dietary components. J. Nutr. 141, 769-776. doi: 10.3945/jn.110. 135657

Walugembe, M., Hsieh, J. C., Koszewski, N. J., Lamont, S. J., Persia, M. E., and Rothschild, M. F. (2015). Effects of dietary fiber on cecal short-chain fatty acid and cecal microbiota of broiler and laying-hen chicks. Poult. Sci. 10, 2351-2359. doi: $10.3382 /$ ps/pev242

Wang, W. W., Jia, H. J., Zhang, H. J., Wang, J., Lv, H. Y., Wu, S. G., et al. (2019). Supplemental plant extracts from flos lonicerae in combination with baikal skullcap attenuate intestinal disruption and modulate gut microbiota in laying hens challenged by Salmonella pullorum. Front. Microbiol. 10:1681. doi: 10.3389/fmicb.2019.01681

Wei, S., Morrison, M., and Yu, Z. (2013). Bacterial census of poultry intestinal microbiome. Poult. Sci. 92, 671-683. doi: 10.3382/ps.2012-02822

Wellington, M. O., Thiessen, R. B., Van Kessel, A. G., and Columbus, D. A (2020). Intestinal health and threonine requirement of growing pigs fed diets containing high dietary fibre and fermentable protein. Animals 11:2055. doi: 10.3390/ani10112055

Zheng, M., Mao, P., Tian, X., Guo, Q., and Meng, L. (2019). Effects of dietary supplementation of alfalfa meal on growth performance, carcass characteristics, meat and egg quality, and intestinal microbiota in Beijing-you chicken. Poult. Sci. 98, 2250-2259. doi: 10.3382/ps/pey550

Zvanych, R., Lukenda, N., Kim, J. J., Li, X., Petrof, E. O., Khan, W. I., et al. (2014) Small molecule immunomodulins from cultures of the human microbiome member Lactobacillus plantarum. J. Antibiot. 67, 85-88. doi: 10.1038/ja.2013. 126

Conflict of Interest: The authors declare that the research was conducted in the absence of any commercial or financial relationships that could be construed as a potential conflict of interest.

Publisher's Note: All claims expressed in this article are solely those of the authors and do not necessarily represent those of their affiliated organizations, or those of the publisher, the editors and the reviewers. Any product that may be evaluated in this article, or claim that may be made by its manufacturer, is not guaranteed or endorsed by the publisher.

Copyright (c) 2021 Hao, Ji, Shen, Wu, Zhang, Tang, Hou and Xie. This is an openaccess article distributed under the terms of the Creative Commons Attribution License (CC BY). The use, distribution or reproduction in other forums is permitted, provided the original author(s) and the copyright owner(s) are credited and that the original publication in this journal is cited, in accordance with accepted academic practice. No use, distribution or reproduction is permitted which does not comply with these terms. 